\title{
Palatal Rugea - an Aid in Clinical Dentistry
}

\author{
Amandeep Bhullar ${ }^{1}$, Raman Preet Kaur ${ }^{1 *}$ and Mamta Sharad Kamat ${ }^{2}$
}

${ }^{1}$ Genesis Institute of Dental Sciences \& Research, Ferozepur, Punjab, India

${ }^{2}$ Modern Dental College, Indore, Madhya Pradesh, India

\begin{abstract}
Among the common methods used in the forensic sciences for confirming the identity are fingerprinting, DNA analysis and dental comparison. In forensic dentistry, the oral cavity plays a very important role because of unique anatomy of teeth. In certain situations, if teeth are lost due to any reason, the most common of which is trauma, then the use of human palatal rugae has been suggested as an alternative method for identification. Palatal Rugae are anatomical wrinkles or folds called 'plica palatine', the irregular connective tissue located on the anterior third of the palate behind the incisive papilla. These are studied for various purposes mainly in fields of anthropology, genetics, orthodontics, prosthodontics, and forensic science.. These are formed by $12^{\text {th }}$ to $14^{\text {th }}$ week of prenatal life and almost remain throughout life. As they are stable landmark, which once formed, do not undergo any changes except in length (due to normal growth) and remain in position throughout person's life. Thus palatoscopy or palatal rugoscopy is used as an aid in clinical dentistry.
\end{abstract}

Keywords: Palatine rugae; Tooth movement; Cleft palate; Ethnic groups; Orthodontic movement

\section{Introduction}

Anatomists always have shown interest in the evolutionary development of the folds of tissue found in the roof of the human mouth-the palatine rugae [1]. Winslow [2] seems to have been the first to describe them, and the earliest illustration of them probably is by Santorini [3]. Palatal rugea, also called plicae palatinae transversae and rugae palatina, refer to thridges on the anterior part of the palatal mucosa, each side of the median palatal raphe and behind the incisive papilla.

A histological study of the development of palatal rugae in mice has shown that they develop as localized regions of epithelial proliferation and thickening even before the elevation of the palatal shelves. Subsequently, fibroblasts and collagen fibres accumulate in the connective tissue beneath the thickened epithelium and then assume a distinctive orientation. The collagen fibres running anteroposteriorly within the curve and in concentric curves across the base of each rugae determine the orientation of the rugae. In human embryos, rugae are relatively prominent and occupy most of the length of the palatal shelves at the time of their elevation [6]. At the $550 \mathrm{~mm}$ stage of embryonic development, there are five to seven rather symmetrically disposed ridges, with the anterior ones beginning at the raph'e, the others more laterally. Towards the end of intra-uterine life, the pattern of rugae becomes less regular, posterior ones disappearing and those anterior become considerably more pronounced and compressed [5]. The rugae are stratified squamous, mainly parakeratinized, epithelium on connective tissue base, similar to adjacent tissue of palate. The reticulin fibre content is very delicate and fibroblasts are different in amount and size from that in adjacent palatal tissue. The development and differentiation of rugae are more advanced in rats than humans and while they are probably involved in oral function in animals, rugae seem to be attenuating in humans [7].

Palatine rugae can be used as internal dental-cast reference points for quantification of tooth migration in cases of orthodontic treatment [8]. For patients who experience difficulty with their speech patterns when acclimating to a new prosthesis, the texture of the rugae in the palatal region of the denture may prove helpful [9]. When traffic accidents, acts of terrorism or mass disasters occur in which it is difficult to identify a person according to fingerprints or dental records, palatine rugae may be an alternative method of identification. The palatine rugae are permanent and unique to each person and can establish identity through discrimination (via casts, tracings or digitized rugae pattern [10]. Palatal rugoscopy was first proposed in 1932, by Spanish investigator named Trobo Hermosa.

\section{Classification of palatine rugae}

The first system of classification was developed by Goria [11] in 1911 and was rudimentary. The rugae pattern was categorized in two ways: specifying the number of rugae and specifying the extent of the rugal zone relative to the teeth. In this system, compound rugae of two or more branches were counted as one, whether they were V- or Y-shaped. Goria further distinguished two types: simple or primitive and more developed. Lysell's [10] classification in 1955 is the most important, and it has been used widely in research involving rugae. It is comprehensive and includes the IP. Rugae are measured in a straight line between the origin and termination and are grouped into three categories:

Primary: 5 millimeters or more;

Secondary: 3 to $5 \mathrm{~mm}$;

Fragmentary: 2 to $3 \mathrm{~mm}$;

Rugae smaller than $2 \mathrm{~mm}$ are disregarded.

This is rather simplified picture of the intricate form that rugae usually present.

Therefore, Thomas and Kotze have further detailed the various

*Corresponding author: Dr. Raman Preet Kaur, Flat no.41, Genesis Institute of Dental Science, Ferozepur-152001, Punjab, India, Tel: 9888910321; E-mail: ramanpreetgrewal@rediffmail.com

Received March 18, 2011; Accepted April 11, 2011; Published April 13, 2011

Citation: Bhullar A, Kaur RP, Kamat MS (2011) Palatal Rugea - an Aid in Clinical Dentistry. J Forensic Res 2:124. doi:10.4172/2157-7145.1000124

Copyright: $\odot 2011$ Bhullar A, et al. This is an open-access article distributed under the terms of the Creative Commons Attribution License, which permits unrestricted use, distribution, and reproduction in any medium, provided the original author and source are credited. 
patterns of primary rugae. These include branched, unified, crosslinked, annular, and papillary, among others.

In 1955, Carrea [13,35] categorized four main types of rugae according to direction. They received roman numerals, while the sequence was indicated according to Arabic numerals and the shape denoted by letters.

The classification of rugea by Basauri $[13,35]$ consists of two groups: simple and compound. These, in turn, are subdivided into 10 types that describe particular shapes: 0 , pointed; 1 , straight; 2 , curved; 3 ,angled; 4, sinuous; 5, circular; 6, Greek; 7, calyxshaped; 8, racket-shaped; 9, branched.The classification by Lima [14] consists of four main types: punctuate, straight, curved and composite.

\section{Clinical significance}

Landmark in orthodontic treatment: Dental casts are threedimensional (3-D) records of malocclusion that have been used successfully during diagnosis and treatment planning for orthodontic patients. The palatine rugae are unique to each patient and are reasonably stable during the patient's growth thus, they may serve as suitable reference points from which the clinician can derive the reference planes necessary for longitudinal cast analysis. Positional changes of posterior teeth in the anteroposterior direction are relevant to the diagnosis and correction of sagittal occlusal abnormalities and arch length discrepancies [15].

Hausser [6] observed orthodontically treated patients and concluded that the lateral edges of the rugae moved forward about onehalf the distance of the migration of the adjacent teeth, while the medial rugae were not affected. In a study of changes occurring in 15 patients who underwent extraction of four premolars, Peavy and Kendrick [16] reported that the lateral ends of the rugae that terminated close to the teeth followed the movement of the teeth in the sagittal plane, but not in the transverse plane. Van der Linden [15] evaluated changes in the position of posterior teeth in relation to palatine rugae in 65 normally growing children (aged 6 to 16 years) and in six orthodontically treated patients. The maximum mean change in distance between the rugae in the anteroposterior plane was $0.41 \mathrm{~mm}$. The authors noted larger movements at both the medial and lateral rugae points in the orthodontically treated patients.

Shriram C Bansode et al. [17] analysed only some changes in the rugae pattern during orthodontic treatment by evaluating the preoperative and postoperative orthodontic casts of 60 patients. They also assessed that the morphology of palatal rugae remains stable throughout life and carefully assessed rugae pattern has definite role in forensic practice [17].

Palatine rugae in speech and palatal prostheses: The significance of palatine rugae in relationship to speech has not been established. These characteristic soft-tissue ridges are present in all primates, and no experimental evidence exists to support their consideration as a speech organ [18]. Palatography has been used to determine the optimum thickness and shape of the palatal surfaces. This approach was developed in a study of phonetics to determine the contact position of the tongue relative to the palate in the production of specific sounds [19] essentially, application of these techniques ensured contact between the tongue and palate during articulation of these sounds. The "s" and "sh" phonemes have received particular attention. Palatography frequently has served as the basis for determining the shape of the anterior palatal vault most conducive to satisfactory sound articulation [20].
Palatal vault shape: The shape of the palatal vault is of particular interest to prosthodontists [21] Snow [22] described the significance of adequate but not excessive contour in the anterior palatal and premolar areas. Central and lateral lisping may develop when the contours of the prosthesis are incorrect. Patients whose speech is sensitive to a changed relationship of the tongue to a palatal prosthesis may require surface texture to orient the tongue. The palatine rugae and the IP often can serve as a cue $[23,24]$. Because the lack of texture on the palatal portion of a complete denture can impede proper articulation, one solution is to add palatine rugae. Unfortunately, the addition of rugae to a prosthesis is not a foolproof method of eliminating speech problems. Landa [25] reported that rugae in dentures are ineffectual or sometimes detrimental to speech if they add unnecessary thickness to the anterior palatal region.

Antero-posterior tooth movement: Hoggan and Sadowsky investigated the use of the palatine rugae as reference points for measuring tooth movement in a manner comparable with cephalometric superimpositions. The authors evaluated the anteroposterior movement of the maxillary first molars and central incisors with the use of two cephalometric variables and six study model variables, and they combined the right and left sides of the palate. The results showed no statistical differences between the mean incisor and molar movement measured cephalometrically and the tooth movement measured relative to the medial and lateral end of the third palatine ruga. Thus, the authors concluded that palatine rugae could be used reliably to assess anteroposterior tooth movements [26].

Palatine rugae in cleft palate patients: Early diagnosis of submucosal cleft palate is important. In children too young to tolerate nasendoscopy and videofluoroscopy, the diagnosis depends on the patient's clinical history and intraoral examination findings. Kratzsch and Opitz investigated the relationship of palatine rugae to points(landmarks) and distances on the cleft palate during the period from birth to the time of early mixed dentition. The authors identified changes in the distances from the lateral palatine rugae points of the first and third rugae to the incisal point, the canine point and the tuberosity line. The results of their study indicated that a comparison of distances from the palatine rugae with distances between equivalent points revealed the changes that occurred in the anterior palate during various stages of orthodontic therapy and growth [27].

Variation of rugae pattern in different ethnic groups: There seems to be a significant association between rugae forms and ethnicity. Kapali and colleagues studied the palatal rugae pattern in Australian Aborigines and whites.

They observed the number, length, shape, direction and unification of rugae. The authors concluded that the mean number of primary rugae in Australian Aborigines was higher than that in whites, although whites had more primary rugae that exceeded $10 \mathrm{~mm}$ in length. The most common shapes in both ethnic groups were wavy and curved forms, while straight and circular forms were least common [4]. Kashima compared the palatine rugae and shape of the hard palate in Japanese and Indian children. They found the following:

Japanese children had more primary rugae than did Indian children, but both groups had the same number of transverse palatine rugae.

The two groups differed with regard to primary rugae shapes, the posterior boundary of the rugal zone, and the number and position of the secondary and fragmentary rugae. The palatal raphae of the Japanese children were wider than those of the Indian children. Both 
groups had many transverse palatine rugae on the left side of the palate. The posterior border of the rugal zone on the left side was shifted farther back than it was on the right side.

There were no significant differences between the two sexes in either group $[28,35]$.

Shetty and colleagues compared the palatine rugae patterns in Indians with those in a Tibetan population. The results of their study showed that males had more rugae on the right side than on the left side in both populations, Indian males had more primary rugae on the left side than did females and vice versa for the Tibetan population, and Indian males had more curved rugae than did Tibetan males [29].

Aparna Paliwal et al. [30] compared the palatal rugae patterns in two different populations in india-Madhya Pradesh and Kerala. The results showed that the straight rugae pattern on the right side of palate in the males was found to be significantly predominant in Madhya Pradesh population,whereas wavy shape was predominant in Keralites;however rugae patterns on the right side of the palate in female subjects exhibited no significant difference [30].

Forensic identification: Establishment of person's identity can be a difficult task in cases of traffic accidents or acts of terrorism or in mass disaster situations. The application of palatal rugae patterns for personal identification was suggested by Allen in 1889. Visual identification, use of dental records and fingerprints and DNA comparisons probably are the most common techniques used in this context, allowing fast and secure reliable identification. However, visual identification and use of fingerprints are limited by postmortem changes associated with time, temperature and humidity. ${ }^{31}$ Although teeth are more durable than other parts of the body, identification via dental records also may prove to be inconclusive, because dental treatment might have been performed between the creation of a dental record and the person's death. Although DNA profiling is accurate, it is expensive and timeconsuming for use in large populations. It is a well-established fact that the rugae pattern is as unique to a human as are his or her fingerprints and it retains its shape throughout life. The anatomical position of the rugae inside the mouth-surrounded by cheeks, lips, tongue, buccal pad of fat, teeth and bone-keeps them well-protected from trauma and high temperatures. Thus, they can be used reliably as a reference landmark during forensic identification. Thomas and van Wyk [33] successfully identified a severely burnt body by comparing the rugae to the pattern on the victim's old denture.

Burn cases: Muthusubramanian et al. [34] did a study to examine the extent of palatine rugae preservation for use as an identification tool in burn victims and cadavers, thus simulating forensic cases of incineration and decomposition. Patients with panfacial third-degree burns (full thickness burns characterized by multicolored denatured layers, dry and insensitive to paininvolving skin, subcutaneoustissues, adnexal structures and nerves and that usually requireskin grafting) were examined within 72 hours after their accident. In addition, human cadavers stored in a mortuary at $5^{\circ} \mathrm{C}$ with 30 to 40 percent relative humidity and kept for a minimum of seven days were assessed for the condition of the palatine rugae. The authors took photographs of the palatine rugae by using a palatal mirror. The study results showed that among the subjects with third-degree panfacial burns, 93 percent of the palatine rugae were normal. The authors observed no changes in the color or surface anatomy of the palatine rugae in 77 percent of the human cadavers. They concluded that the palatine rugae could be used as a reference landmark during forensic identification of an individual [34].

\section{Conclusion}

Located in the anterior half of the roof of the mouth, palatal rugea serve as a reference landmark in various dental treatment modalities and could be used in the identification of submucosal clefts. Palatine rugae can be used to assess the amount of anteroposterior tooth movement, because they remain stable during a person's life. Moreover, the results of several studies show a significant association between rugae forms and different races. Palatine rugae are unique to individual can therefore be used for individual identification in forensic odontology.

\section{References}

1. Salzman JA, Lysell $L$ (1955) Plicae palatinae transversae and papillae incisiva in man; a morphologic and genetic study. Am J Orthod 41: 879-880.

2. Winslow JB (1732) Exposition Anatomique de la structure du corps humain.

3. Santorini JD (1775) Septemdecim Tabulae.

4. Kapali S, Townsend G, Richards L, Parish T (1997) Palatal Rugea in Australian Aborigenes and Caucasians. Aust Dent J 42: 129-133.

5. Luke DA (1988) Development and growth of palatal rugae in the mouse. Acta Anat 133: 41-44.

6. Hauser G, Daponte A, Roberts MJ (1989) Palatal rugae. J Anat 165: 237-249.

7. Thomas CJ, Rossouw RJ (1991) The early development of palatal rugae in the rat. Aust Dent J 36: 342-348.

8. Simmons JD, Moore RN, Erickson LC (1987) A longitudinal study of anteroposterior growth changes in the palatine rugae J Dent Res 66: 1512 1515.

9. Gitto CA, Esposito SJ, Draper JM (1999) A simple method of adding palatal rugae to a complete denture. J Prosthet Dent 81: 237-239.

10. English WR, Robison SF, Summitt JB, Oesterle LJ, Brannon RB, et al. (1988) Individuality of human palatal rugae. J Forensic Sci 33: 718-726.

11. Goria C (1911) Le rughe del palato in speciale rapporto coll anthropologia criminale e la psichiatria.

12. Carrea JU (1955) Gaumenfalten-Fotostenogramme, ein neues identfizierungsverfahren [Fotostenograms of palate folds, a new identification technic]. Dtsch Zahnarztl Z 10: 11-17.

13. Basauri C (1961) Forensic odontology and identification 16: 45.

14. Lima OC (1968) Rugoscopia [Rugoscopy (Correia Lima's process)] RevBrasMed 25: 806-807.

15. van der Linden FP (1978) Changes in the position of posterior teeth in relation to ruga points. Am J Orthod 74: 142-161.

16. Peavy DC Jr, Kendrick GS (1967) The effects of tooth movement on the palatine rugae J Prosthet Dent 18: 536-542.

17. Bansode SC, Kulkarni MM (2009) Importance of palatal rugae in individual identification. J Forensic Dent Sci 2: 27-31.

18. Chierici G, Lawson L (1973) Clinical speech considerations inprosthodontics: perspectives of the prosthodontist and speech pathologist. J Prosthet Dent 29 : 29-39.

19. Moses ER Jr, Speech QJ (1940) A brief history of palatography. 26: 615-625.

20. Bloomer HH, JADA (1943) A palatograph for contour mapping of the palate. 30: 1053-1057.

21. Silverman MM (1967) The whistle and swish sound in denture patients $\mathrm{J}$ Prosthet Dent 17: 144-148.

22. Snow GB (1889) The proper configuration of the lingual surfaces of dental plates. 20: 51-54.

23. Palmer JM (1979) Structural changes for speech improvement in complete upper denture fabrication. J Prosthet Dent 41: 507-510.

24. Pound E (1951) Esthetic dentures and their phonetic values J Prosthet Dent 1: $98-111$. 
25. Landa J (1935) The importance of phonetics in full denture prosthetics. Dent Dig 41: 154-160

26. Hoggan BR, Sadowsky C (2001) The use of palatal rugae for the assessment of anteroposterior tooth movements. Am J Orthod Dentofacial Orthop 119: 482 488.

27. Kratzsch H, Opitz CJ (2000) Investigations on the palatal rugae pattern in cleft patients, part II: changes in the distances from the palatal rugae to maxillary points. Orofac Orthop 61: 421-431.

28. Kashima K (1990) [Comparative study of the palatal rugae and shape of the hard palatal in Japanese and Indian children]. Aichi Gakuin Daigaku Shigakkai Shi 28: 295-320.

29. Shetty SK, Kalia S, Patil K, Mahima VG (2005) Palatal rugae pattern in Mysorean and Tibetan populations. 16: 51-55.
30. Paliwal A, Wangari S, Pawani R (2010) Palatal Rugoscopy:Establishing Identity. J Forensic Dent Sci 2: 27-31.

31. Morlang WM (1982) Forensic dentistry. Aviat Space Environ Med 53: 27-34.

32. Lysell L (1955) Plicae palatinae transversae and papilla incisiva in man: a morphologic and genetic study. Acta Odontol Scand 13: 5-137.

33. Thomas CJ, Van Wyk CW (1987) Elastic fibre and hyaluronic acid in the core of human palatal rugae. J Biol Buccale 15: 171-174.

34. Muthusubramanian M, Limson KS, Julian RJ (2005) Analysis of rugae in burn victims and cadavers to simulate rugae identification in cases of incineration and decomposition. Forensic Odontostomatol 23: 26-29.

35. Patil MS (2008) Clinical dentistry. 139: 1471-1478. 